\title{
METHOD OF INDIRECT DETERMINATION OF THERMAL LOAD PARAMETER
}

\author{
Yu. Baidak ${ }^{1, *}$, I. Vereitina ${ }^{1}$
}

\section{ABSTRACT}

An indirect method for determining the effectiveness of the transformers operating in a distribution network with variable load schedules, according to the parameter of their thermal load as a ratio of heat accumulated by transformer oil to the maximum permissible heat is proposed provided the thermal stability of the insulation. a mathematical model is developed which allows establishing analytically the forced temperature value and time invariable of heating or cooling on the section of the time dependence of the oil temperature, as well as an algorithm for calculating the amount of heat accumulated by the oil and the coefficient of heat transfer from the oil to the transformer tank is compiled. approbation of the indirect method indicates that the divergence between the transformer oil heating time invariable which was obtained by solving the differential equation of thermal balance and calculated using the part of temperature dependence of its heating for two minutes, is $3.6 \%$, and at determination of the forced temperature value $-0.8 \%$.

Keywords: Transformer, Thermal Subsystem, Energy Efficiency, Thermal Load, Timing Diagrams of Temperatures

\section{INTRODUCTION}

Transformers involved in power distribution networks are designed and manufactured according to the minimum of the additive criterion of the combined costs of production and during operation, since these components are considered by manufacturers and consumers as the most influential in the composition of power efficiency.

Unfortunately, not all technical requirements that a transformer must meet, are satisfied, as well as those partial criteria that are taken into consideration are reasonable technically and economically. As a result, material

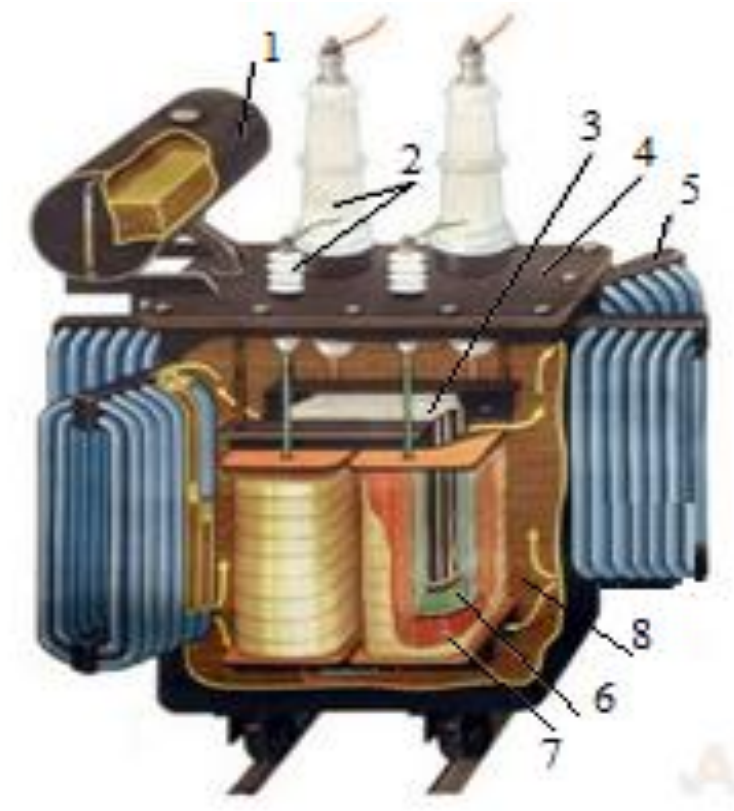

Figure 1. Single-phase transformer of distribution networks in the cross-section, in which: 1 - expander tank, 2 - insulators of windings ends, 3 - magnetic core, 4 - tank cover, 5 - natural cooling radiators, 6,7 low and high voltage windings, 8 - transformer oil

and human costs inherent in the transformer is not used in full, including the parameter of its load. 
With regard to the prospects for the additional design and development of existing transformers, the availability of opportunities for their economic justification, modification or development of new technical requirements, they are considered based on the actual analysis of the most important parameter of the present cost estimation of performance. Typically, a feasibility study is performed on the existing equipment and on the basis of case-studies; although it is known that the greatest effect can be achieved at the last stage. This approach allows reconciling the functional resources of the transformer with the actual operating conditions. The modern industry has accumulated considerable experience in the development and production of transformers, which provide economic effect and economical transformation of energy, but provided they work continuously and at full capacity.

The parameters of optimization in them are: the diameter of the core of the magnetic circuit, the current density in the windings, temperature and dimensional parameters, but among them there is still no parameter that would take into account the level of load of the transformer during operation.

In the case of power outages and rolling blackouts or temporary overload of some of the transformers it becomes a significant factor.

Since the loss of electrical energy in the elements of the transformer constructions leads to their heating, and they are dependent on the load, the transformer itself can be regarded as a thermal system, and the heating temperature is an indirect sign of its load, which is adequate to determine the operational efficiency index - the thermal load.

\section{THERMAL LOAD PARAMETER SIMULATION METHOD}

Transformation of electric energy into a useful work is always accompanied by its partial loss with transformation into heat [1]. Heat transfer $Q$ from the surface of the body is carried out simultaneously by all its types. Therefore, when calculating the coefficient of heat transfer, a generalized heat transfer coefficient (heat remove) $k_{T}$ is used.

For the case of a stable mode, when all $P$ power losses are allocated to the environment $Q=P$, the heat remove is calculated from the Newton equation $Q=k_{T} F\left(\theta-\theta_{0}\right)=k_{T} F \tau_{\mathrm{y}}$ or in the form of the Ohm's law $Q=\frac{\tau_{\mathrm{y}}}{R_{\mathrm{T}}}$ in which $R_{\mathrm{T}}=\frac{1}{k_{\mathrm{T}} F}$ is the thermal resistance, $\tau_{\mathrm{y}}$ is the excess of the set temperature. Consequently, the main problem in installing heat transfer is to determine the heat transfer coefficient from the surfaces, which depends on many factors, and in liquids it is also determined by convective heat exchange. In the design practice the coefficients of heat transfer are determined collectively, with the use of empirical coefficients [2] [3].

Therefore, solving the problem of refining the value of the heat transfer coefficient, at any stage of its application, remains relevant [4]. It is known that the most substantial sources of heat in the transformer are: the steel magnet wire - with loss of idle speed $P_{0}$ and conductor windings materials - with losses of short circuit $P_{k}$. According to the design of the transformer, Figure 1, the magnetic core and the windings of the active part are in the cooling medium - transformer oil, which is to be the conductor of the heat flow to the tank casing, and from it into the environment. Consequently, its temperature should be initiated to establish a heat load.

The complete solution of the inhomogeneous differential equation of the thermal balance compiled for the cooling oil medium and such that is located inside the tank whose surface is in contact with the environment at a temperature $\theta_{0}$ and absorbs the heat from the magnetic core and the transformer windings, is presented in [5] in the form

$$
\theta_{\text {oil }}=\theta_{\text {oil.pr }}\left(1-e^{\frac{1}{-T_{\text {oil }}} \cdot t}\right)+\theta_{0} e^{\frac{1}{-T_{\text {oil }}} \cdot t}
$$

where $\theta_{\text {oil.pr }}$ - constant or forced temperature of the oil, which it reaches during the long operation of the transformer without changing the load, that is, at $\mathrm{t}=\infty$.

Figure 2 shows the time dependence of the oil heating temperature for (1) at $\theta_{0}=0$ (continuous line) when the transformer is switched on for a nominal load within 1 hour 30 minutes, as well as its normalized value according to Standards (interruptible line). Exceeding of the rationed value of $\theta_{S t}=60^{\circ} \mathrm{C}$ adversely affects the dielectric resistance of winding insulation and is unacceptable as for its heat resistance class. The given dependence can also be obtained experimentally, according to the indications of the thermometer, which is set at the reference point of temperature measuring of the upper oil layer under the tank cover. 


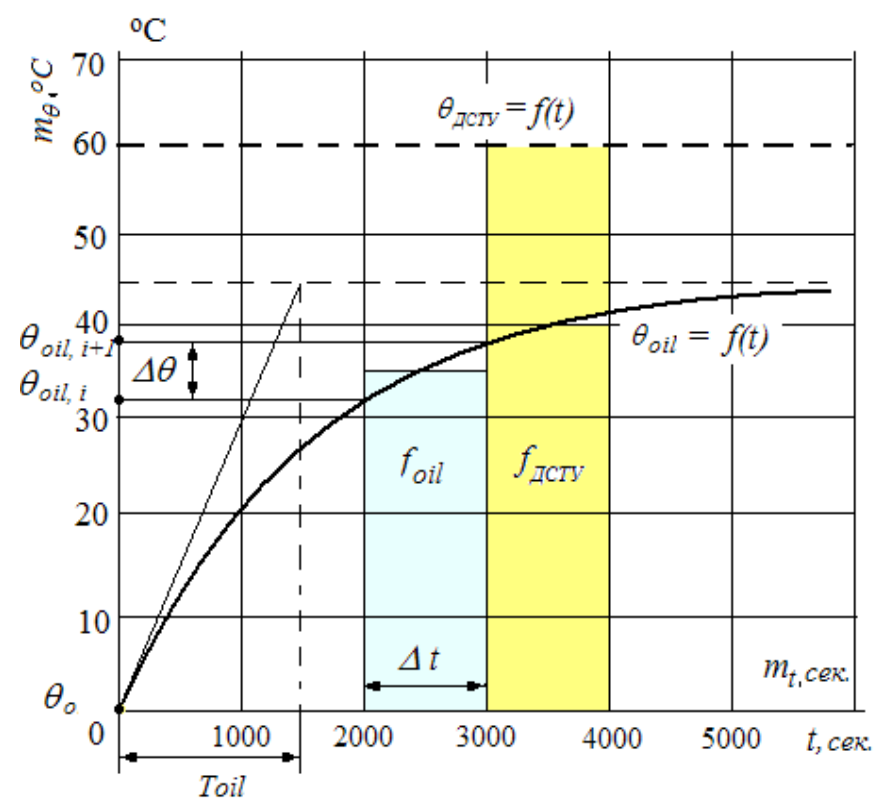

Figure 2. Time diagram of temperature for determining the equivalent of heat flow $f_{\text {oil }} m_{t} m_{\theta}$ and $f_{S t} m_{t} m_{\theta}$

If, for the purpose of transformer thermal load determining, the ratio of the amount of heat absorbed by the oil by heat transfer and convection from the heat sources to its permissible value at the temperature established by the Standard will be applied, then, during the transformer operation time, even with a variable load, they are proportional to the areas of the surfaces formed in the lower part of the surface by the environment temperature $\theta_{0}$, and in the upper part by the variable temperature values of the upper layer of oil $\theta_{\text {oil }}$ and, consequently, by the constant temperature according to the $\theta_{S t}$. In the general case, we have the equivalents of heat fluxes $f_{\text {oil }} m_{t} m_{\theta}, f_{S t} m_{t} m_{\theta}$, in which $m_{t}, m_{\theta}$ are the scales on the abscissa and ordinate axes in the time charts, respectively (in $\mathrm{h} / \mathrm{mm})$ and $\left({ }^{\circ} \mathrm{C} / \mathrm{mm}\right) ; f_{\text {oil }}$ and $f_{S t^{-}}$surfaces areas $\left(\mathrm{mm}^{2}\right)$ formed by temperature and time in the coordinate system $\theta_{\text {oil }}=f(t)$. Thus, the actual amount of heat removed by the transformer oil and its permissible value will be

$$
\begin{aligned}
& Q_{o i l}=k_{T} F_{f r} \int_{0}^{t}\left(\theta_{o i l}-\theta_{0}\right) e^{\frac{1}{-T_{o i l}} \cdot t} d t=k_{T} F_{f r} \sum_{i=1}^{n} f_{\text {oil }, i} m_{t} m_{\theta} \\
& Q_{S t}=k_{T} F_{f r} \int_{0}^{t}\left(\theta_{\text {дSt }}-\theta_{0}\right) d t=k_{T} F_{f r} \sum_{i=1}^{n} f_{S t} m_{t} m_{\theta} .
\end{aligned}
$$

The construction of an algorithm for heat amount calculation according to the oil temperature monitoring data and the known time dependence of the change in its temperature during heating at the transformer load activation, requires a numerical solution of the equations (2) according to the experimentally obtained fraction of the temporal temperature diagram and the presence of known values of the working time $t$ from the beginning of integration; the time constant of the transitional heating process $-T_{\text {oil }}$; the coefficient of heat transfer and the heat flow to the inner surface of the tank- $\mathrm{k}_{\mathrm{T}}$; the total surface area of tank cooling $-F_{f r}$

The invariable of integration can be calculated analytically by solving the equation of the thermal balance compiled for the oil in the cooling system or determined graphically by the experimentally obtained dependence $\theta_{\text {oil }}=f(t)$. Such approach in some way complicates the construction of a technical device - a tester of thermal load and, therefore, requires an analytical solution. 


\section{THE ALGORITHM OF THE THERMAL LOAD PARAMETER MODEL}

The reason for thermal load parameter mathematical model creation is the presence of a section of the time dependence of the oil temperature in the cooling system, which can be obtained in any way. To determine the forced temperature of the transformer oil $\theta_{\text {oil.forced }}$ in (1) we use the indirect method. Initially, we establish the dependence of the oil temperature change on the time of the transition process progressing, that is, $\frac{\Delta \theta_{\text {oil }}}{\Delta t}=$ $f(t)$. For example, after the differentiation of equation (1) we obtain

$$
\frac{d \theta_{\text {oil }}}{d t}=-\frac{1}{T_{\text {oil }}}\left(\theta_{\text {oil.forced }} e^{-\frac{t}{T_{\text {oil }}}}-\theta_{0} e^{-\frac{t}{T_{\text {oil }}}}\right)
$$

in which, from the same expression (1),

$$
\theta_{\text {oil.forced }} e^{-\frac{t}{T_{\text {oil }}}}=\theta_{\text {oilforced }}-\theta_{\text {oil }}-\theta_{0} e^{-\frac{t}{T_{\text {oil }}}}
$$

By substituting (4) into (3), we have

$$
-T_{\text {oil }} \frac{d \theta_{\text {oil }}}{d t}=\theta_{\text {oilforced }}-\theta_{\text {oil }}+\theta_{0} e^{-\frac{t}{T_{\text {oil }}}}-\theta_{0} e^{-\frac{t}{T_{\text {oil }}}}=\theta_{\text {oil.forced }}-\theta_{\text {oil }},
$$

from where the oil temperature will be determined by the linear equation

$$
\theta_{\text {oil }}=\theta_{\text {oil.forced }}-T_{\text {oil }} \frac{d \theta_{\text {oil }}}{d t}
$$

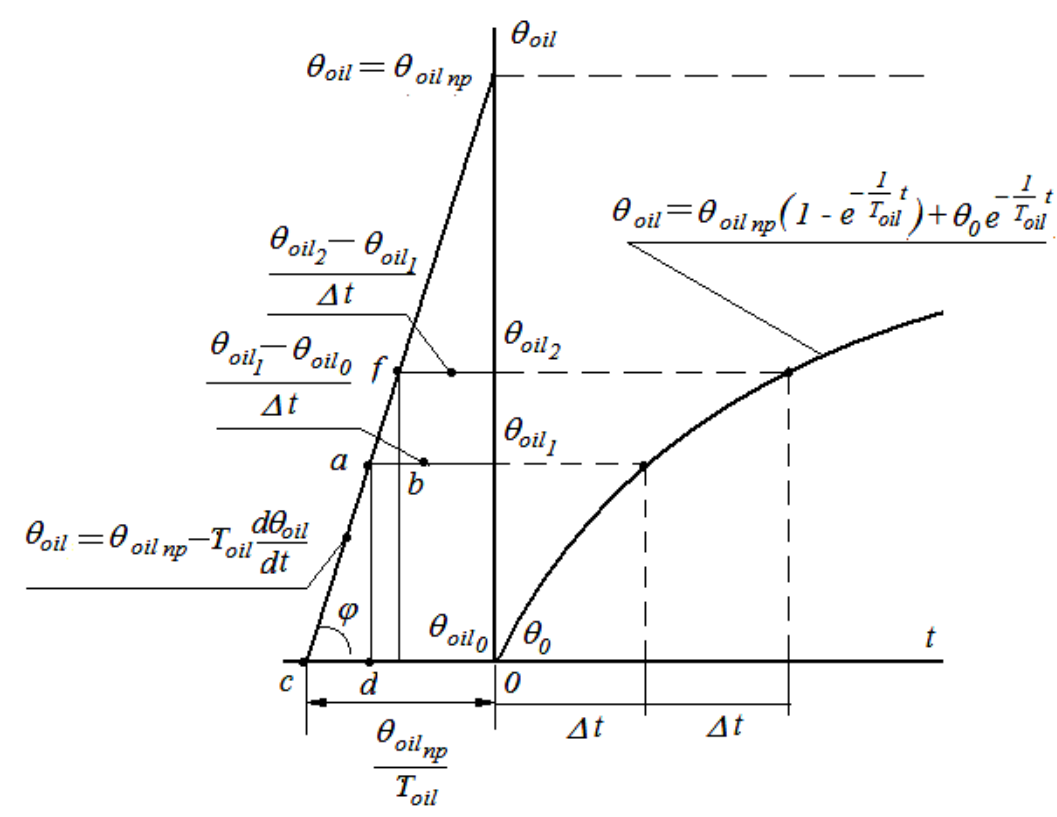

Figure 3. To the calculation of the values of the forced temperature of the oil heating and its time invariable by indirect method

Figure 3 shows the fraction of the time dependence of the oil heating temperature $\theta_{\text {oil }, i}(t)$, constructed on the basis of numerical calculations of the equation of its thermal balance and on which the linear dependence (5) was constructed on the left to determine the forced value of oil temperature and the time invariable at the load of the transformer. As follows from the drawing, the angle of inclination of the dependence (5) is invariable and can be defined from the triangle $a b f$ as follows: 
- a section of temperature change rate after the second measurement of it

$$
\overline{a b}=\frac{\theta_{o i l, 1}-\theta_{o i l, 0}}{\Delta t}-\frac{\theta_{o i l, 2}-\theta_{o i l, 1}}{\Delta t}=\frac{2 \theta_{o i l, 1}-\theta_{o i l, 0}-\theta_{o i l, 2}}{\Delta t} ;
$$

- the angle of inclination of linear dependence (5)

$$
\varphi=\tan ^{-1} \frac{\theta_{o i l, 2}-\theta_{o i l, 1}}{\overline{a b}}
$$

- the period of temperature change after the first measurement

$$
\overline{c d}=\frac{\theta_{o i l, 1}-\theta_{o i l, 0}}{\tan \varphi}=\frac{\left(\theta_{o i l, 1}-\theta_{o i l, 0}\right)}{\left(\theta_{o i l, 2}-\theta_{o i l, 1}\right)} \overline{a b}
$$

- the ratio of the forced temperature to the heating time variable at the beginning of the transformer load

$$
\overline{c 0}=\frac{\theta_{\text {oil.forced }}}{T_{\text {oil }}}=\frac{\theta_{o i l, 1}-\theta_{o i l, 0}}{\Delta t}+\overline{c d}
$$

- tangent of linear dependence (5) inclination angle from (11)

$$
\tan \varphi=\frac{\theta_{\text {oil.forced }}-\theta_{\text {oil }, 0}}{\frac{\theta_{\text {oil.forced }}}{T_{\text {oil }}}}
$$

where the forced oil temperature will be

$$
\theta_{\text {oil.forced }}=\theta_{\text {oil }, 0}+\frac{\theta_{\text {oil.forced }}}{T_{\text {oil }}} \tan \varphi
$$

and the heating time invariable from the expression (5)

$$
T_{o i l}=\frac{\theta_{\text {oil.forced }}-\theta_{\text {oil }}}{\frac{\Delta \theta_{\text {oil }}}{\Delta t}}
$$

As a parameter of thermal load (TL) it is possible to apply the ratio of the heat flow to the oil at the actual load of the transformer to its permissible value according to the Standard

$$
T L=Q_{o i l} / Q_{S t},
$$

where

$$
Q_{S t}=k_{T} F_{f r} \int_{0}^{t}\left(\theta_{S t}-\theta_{0}\right) d t=k_{T} F_{f r}\left(\theta_{S t}-\theta_{0}\right) t
$$

\section{APPROBATION OF THE HEAT LOAD DEFINITION METHOD}

The calculation of the thermal load parameter must begin immediately after the transformer has been switched on to the mains and carried out at identical time intervals $\Delta t=\left(t_{i}-t_{i-1}\right)$, Figure 2 , a countdown of the temperature values at the beginning of $\theta_{\text {oil }, i=0}=\theta_{0}$ and at the end of each time interval $\theta_{\text {oil }, i+1}$.

According to current temperatures for each time interval $i=1, \ldots t / \Delta t$ are calculated: - temperature average value (step approximation on time interval $\Delta t$, Figure 2) 


$$
\Delta \theta_{\text {oil.mid }}=0,5\left(\theta_{\text {oil. } i}+\theta_{\text {oil. } i-1}\right) ;
$$

- the equivalent of the heat flow amount, which oil gives to the transformer tank casing in one time interval

$$
f_{\frac{\text { oil }}{s t}, i} m_{t} m_{\theta}=\frac{\Delta t}{3600} \Delta \theta_{\text {oil.mid }}+f_{\frac{\text { oil }}{s t}, i-1} m_{t} m_{\theta}
$$

where $f_{\frac{o i l}{s t}, i=0} m_{t} m_{\theta}=0$, and $i=1,2, \ldots, \frac{t}{\Delta t}$;

- using expressions $(8 \ldots 14)$ the forced value of the oil temperature and the time invariable of the temperature change transition process can be numerically calculated:

$$
T_{o i l}=\left(\theta_{\text {oil.forced }}-\theta_{\text {oil }, i}\right) \frac{\Delta t_{i}}{\left(\theta_{o i l, i}-\theta_{o i l, i-1}\right)}
$$

- coefficient of heat transfer from oil to the tank

$$
k_{T}=\frac{c_{i} G_{o i l}}{T_{o i l} F_{f r}},
$$

where $c_{i}$ is the specific heat of oil, its approximation, as a linear function, has the form of equation (20), $G_{\text {oil }}$ the weight of oil in the tank and radiators (is indicated on the plate of the investigated transformer)

$$
c=1,549+0,00593 \theta_{o i l, i}
$$

- amount of heat that is transferred by oil from the active part of the transformer to the tank casing during the time $t$ and according to the equation (2)

$$
Q_{o i l}=-T_{o i l} k_{T} F_{f r} \sum_{i=1}^{n} f_{o i l, i} m_{t} m_{\theta} ;
$$

- allowable amount of heat that can be transferred from the active part of the transformer to the tank casing during the operation time $t$ and according to the class of applied insulation

$$
Q_{S t}=t k_{T} F_{f r}\left(\theta_{S t}-\theta_{0}\right) .
$$

The coefficient of thermal load is calculated as the ratio of the amount of heat removed from the useful volume of the tank into the environment to the allowable amount of heat over the test time by the equation (15). The higher the value of the obtained parameter, the more efficient the transformer is used.

For approbation of the indirect method of thermal load determination, a transformer of TM $1000 \mathrm{kVA} 10 / 0.4 \mathrm{kV}$ type is considered. Obtained for it in [5] numerical calculations are: forced temperature of the cooling oil $\theta_{\text {oil.forced }}=40,3^{\circ} \mathrm{C}$; time invariable $-T_{\text {oil }}=870,8$ сек. As a result of calculations, the time chart of the transformer under consideration oil heating temperature is drawn in Figure 4. Additionally, it is known that the weight of the transformer oil according to the catalog is $G_{o i l}=700 \mathrm{~kg}$, and the design value of the area of the cooling surface of the tank with radiators (SB) per phase is $S B=18 \mathrm{~m}^{2}$.

In accordance with the algorithm of the transformer thermal load parameter model and based on the table values of time and temperature of the oil in Figure 4, for intervals $i=2,3$, we have: - the average value of temperature, with the degree approximation at the second interval of time $\Delta t=50 \mathrm{sec}$.

$$
\Delta \theta_{\text {oil.mid }}=0,5(4,372+2,249)=3,288^{\circ} \mathrm{C} ;
$$

- temperature change rate after the second measurement 
Journal of Thermal Engineering, Research Article, Vol. 5, No. 2, Special Issue 9, pp. 84-92, February, 2019

$$
\overline{a b}=\frac{2 \theta_{o i l, 1}-\theta_{o i l, 0}-\theta_{o i l, 2}}{\Delta t}=\frac{2 * 2,249-0-4,372}{50}=0,00252 \frac{{ }^{\circ} \mathrm{C}}{\mathrm{sec}} ;
$$

- the angle of inclination of linear dependence

$$
\varphi=\tan ^{-1} \frac{\theta_{o i l, 2}-\theta_{o i l, 1}}{\overline{a b}}=\tan ^{-1} \frac{4,372-2,249}{0,00252}=89,93^{0}
$$

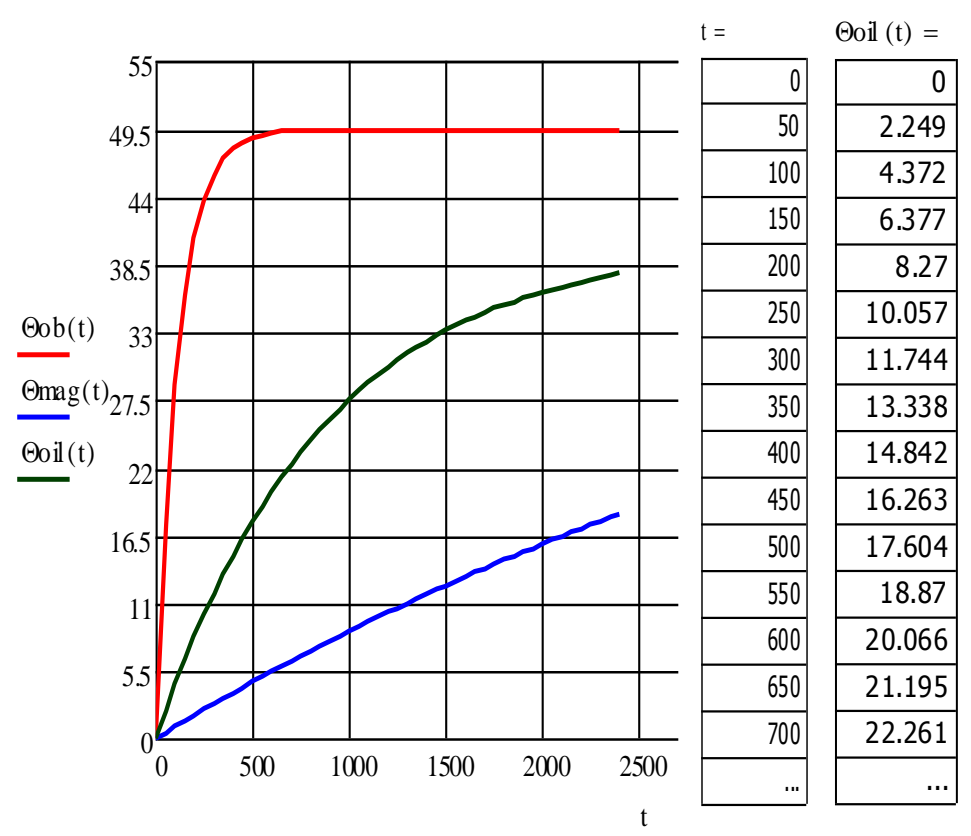

Figure 4. Time chart of heating temperatures for a period of 40 minutes after switching on the transformer on full power (windings - higher, magnetic core - lower, oil - average line)

- change in temperature after the first measurement

$$
\overline{c d}=\frac{\theta_{o i l, 1}-\theta_{o i l, 0}}{\tan \varphi}=\frac{2,249-0}{818,5}=0,00275
$$

- the ratio of the forced temperature to the heating time invariable

$$
\frac{\theta_{\text {oil. } f o r c e d}}{T_{\text {oil }}}=\frac{\theta_{\text {oil }, 1}-\theta_{\text {oil }, 0}}{\Delta t}+\overline{c d}=\frac{2,249-0}{50}+0,00275=0,04773 \frac{{ }^{\circ} \mathrm{C}}{\text { sec. }}
$$

- forced oil temperature value

$$
\theta_{\text {oil.forced }}=\theta_{\text {oil }, 0}+\frac{\theta_{\text {oil.forced }}}{T_{\text {oil }}} \tan \varphi=0+0,04773 * 818,5=39,96^{\circ} \mathrm{C},
$$

- oil heating time invariable

$$
T_{\text {oil }}=\frac{\theta_{\text {oil.forced }}-\theta_{\text {oil }}}{\frac{\Delta \theta_{\text {oil }}}{\Delta t}}=\frac{39,96-2,249}{0,04498}=838,4 \text { sec. } ;
$$

- specific heat of oil 
Journal of Thermal Engineering, Research Article, Vol. 5, No. 2, Special Issue 9, pp. 84-92, February, 2019

$$
c=1,549+0,00593 * 2,249=1,562\left(\frac{k W * s e c}{k g^{\circ} \mathrm{C}}\right)
$$

- coefficient of heat transfer from oil to the tank

$$
k_{T}=\frac{c_{i} G_{o i l}}{T_{o i l} F_{f r}}=\frac{1562 * 700}{838,4 *(18 * 3)}=24,14\left(\frac{W}{m^{2}{ }^{\circ}}\right) ;
$$

- the equivalent of the amount of heat flow, which oil is transferred to the tank during time $t=700 \mathrm{sec}$.

$$
\begin{aligned}
& \sum_{i=1}^{14} \frac{f_{\text {oil }, i} m_{t} m_{\theta}}{3600}=\frac{50}{3600}(1,1245+3,288+5,3745+7,3235+9,1635+10,9005+ \\
& 12,541+14,115+15,5525+16,9335+18,237+19,468+20,6305+21,728)=50 * \\
& 176,38=2,45\left({ }^{\circ} \mathrm{C} \cdot h\right),
\end{aligned}
$$

where

$$
\frac{f_{\frac{o i l}{S t}, i=0} m_{t} m_{\theta}}{3600}=0 \text {, and } i=1,2, \ldots,\left(\frac{700}{50}=14\right)
$$

- amount of heat transferred by oil from the active part of the transformer to the tank surface for $t=700 \mathrm{sec}$. (operating time is determined by the limited table data given in Figure 4)

$$
Q_{o i l}=k_{T} F_{f r} \sum_{i=1}^{14} f_{o i l, i} m_{t} m_{\theta}==24,14 *(3 * 18) * 2,45=3193,7(W \cdot h) ;
$$

- the allowable amount of heat that can be carried by the oil to the transformer tank during the operating time $t=$ $700 \mathrm{sec}$. according to the class of applied winding insulation

$$
Q_{S t}=k_{T} F_{f r} \sum_{i=1}^{14} \frac{f_{S t, i} m_{t} m_{\theta}}{3600}=24,14 *(3 * 18) * 11,67=15212,5 \quad(W \cdot h)
$$

- thermal load parameter (TL)

$$
\mathrm{T} L=\frac{Q_{\text {oil }}}{Q_{S t}} 100=\frac{3193,7}{15212,5} 100=21 \%
$$

Due to the known power losses in the phase of windings LV (low voltage) $P_{1 L V}=1736 \mathrm{~W}$, HV (high voltage) $P_{1 H V}=2114 \mathrm{~W}$ and in the core of the magnetic system $P_{20}=467 \mathrm{~W}$, and those which are placed in the basis of calculating of time dependences of the transformer temperature, given on Figure 4, and in general are

$$
\Delta P=P_{1 L V}+P_{1 H V}+P_{20}=4317 W
$$

we obtain the efficiency of the applied system of transformer cooling within one hour of its operation after switching on

$$
\eta_{c o}=\frac{Q_{o i l}}{\Delta P \cdot t} 100=\frac{3193,7}{4317 \cdot 1} 100=74 \%
$$

\section{CONCLUSIONS}

1. An indirect method for determining the effectiveness of the transformers operating in a distribution network with variable load schedules, according to the parameter of their thermal load as a ratio of heat accumulated by transformer oil to the maximum permissible heat is proposed provided the thermal stability of the insulation.

2. A mathematical model is developed which allows establishing analytically the forced temperature 
value and time invariable of heating or cooling on the section of the time dependence of the oil temperature, as well as an algorithm for calculating the amount of heat accumulated by the oil and the coefficient of heat transfer from the oil to the transformer tank is compiled.

3. Approbation of the indirect method indicates that the divergence between the transformer oil heating time invariable which was obtained by solving the differential equation of thermal balance and calculated using the part of temperature dependence of its heating for two minutes, is $3.6 \%$, and at determination of the forced temperature value $-0.8 \%$

\section{REFERENCES}

[1] Yildiran, R., Atas, S., Kahraman, M. (2016). Design and performance analysis of a district heating system utilizing waste heat of a thermal power plant. Journal of Thermal Engineering, Vol. 2, No. 5, 940-954.

[2] Lienhard, John H. IV; Lienhard, John H. V (2008). A Heat Transfer Textbook (3rd ed.). Cambridge, Massachusetts: Phlogiston Press. ISBN 978-0-9713835-3-1.

[3] Incropera, F. et al. (2012). Fundamentals of heat and mass transfer (7th ed.). Wiley. 2012. 710 p. ISBN 978-0470-64615-1

[4] Maurya, R.S, Singh, S.(2017).Numerical investigation of isothermal flow around impingement plates in a shell and tube exchanger. Journal of Thermal Engineering, Vol. 3, No. 5. 1442-1452.

[5] Matukhno V., Baidak Yu., Tomlein P. (2016). Thermal subsystem of voltage distribution transformer. Refrigeration engineering and technology. Vol 52(6) URL: file:///C:/Users/User/Downloads/477-1152-1-PB (4).pdf 